\title{
Reformas estructurales y Re-reformas de pensiones, y sus efectos en el bienestar social: el caso de Chile
}

\author{
Carmelo MeSA-LAGO \\ Universidad de Pittsburgh \\ (Pensilvania-USA) \\ cmesa@usa.net
}

Recibido: 27-05-2014

Aceptado: 01-10-2015

\section{Resumen}

El artículo primero resume las reformas estructurales de pensiones (privatización total o parcial) en América Latina y Europa Central y Oriental, identificando sus ventajas y desventajas, así como del emergente proceso internacional de re-reforma de pensiones con un mayor rol del Estado. Segundo, escoge a Chile como caso de estudio, por ser pionero en el mundo en ambas reformas; describe las características y efectos en el bienestar social, tanto de la reforma estructural de 1981 como de la re-reforma de 2008. Dichos efectos se evalúan basados en diez principios de seguridad social de la Organización Internacional del Trabajo (OIT): 1) diálogo social para aprobar la reforma, 2) cobertura universal de la población, 3) trato uniforme de los asegurados, 4) solidaridad social, 5) equidad de género, 6) suficiencia en las prestaciones, 7) eficiencia y costo administrativo razonable, 8) participación social en la gestión del sistema, 9) rol del Estado y supervisión, y 10) sostenibilidad financiera. Tercero, resume las ventajas y desventajas-retos de la re-reforma e informa del debate actual sobre reformas para el futuro.

Palabras clave: reformas de pensiones estructurales; privatización; modelos; re-reformas de pensiones; efectos en bienestar social; principios de seguridad social; Chile. 


\title{
Pension Structural Reforms, Re-reforms and their Effects on Social Welfare: The Case of Chile
}

\begin{abstract}
This article first summarizes the structural reforms of pensions (total or partial privatization) in Latin America and Central and Eastern Europe, identifying their advantages and disadvantages, and does the same with the international process of re-reforms of pensions with a greater role of the state. Second, chooses Chile as a case study, as a world pioneer in both types of reforms; describes their characteristics and effects on social welfare of the structural reform of 1981 and the re-reform of 2008. Such effects are evaluated based on ten basic principles of social security from the International Labour Office (ILO): 1) social dialogue to approve the reforms, 2) universal coverage of the population, 3) equal treatment of insured persons, 4) social solidarity, 5) gender equity, 6) sufficiency of benefits, 7) efficiency and reasonable administrative cost, 8) social participation in the management of the system, 9) role of the state and supervision, and 10) financial sustainability. Third, it summarizes the advantages and disadvantages-challenges of the re-reform and informs on the current debate for further reforms.
\end{abstract}

Keywords: Structural Pension Reforms; Privatization; Models; Re-reform of Privatized Pensions; Effects on Social Welfare; Social Security Principles; Chile.

\section{Referencia normalizada}

Mesa-LaGo, C. (2015): "Reformas estructurales y Re-reformas de pensiones, y sus efectos en el bienestar social: el caso de Chile", Política y Sociedad, 52 (3), pp. 719-739.

Sumario: 1. Introducción; 2. El Caso de Chile. 3. Conclusiones: ventajas y problemas / retos de la re-reforma. 4. Epílogo. 5. Bibliografía. 


\section{Introducción}

En las tres últimas décadas "reformas estructurales" privatizaron total o parcialmente las pensiones "publicas" de seguridad social en el mundo.1 Ellas cambiaron los sistemas "públicos" tipificados por prestación definida, financiación por reparto o colectiva parcial y administración pública (a través del seguro social), hacia sistemas "privados" tipificados por cotización definida, capitalización plena con cuentas individuales, $\mathrm{y}$ administración privada con fines de lucro. Comenzando con Chile en 1981, el pionero, y terminando con Rumanía en 2008, 23 países realizaron algún tipo de privatización de pensiones, 13 de ellos en América Latina y diez en Europa Central y Oriental.2 El Banco Mundial (1994) publicó un libro que promovió las reformas y prestó apoyo financiero y técnico a muchos países. Reformas parciales fueron aplicadas en Europa Occidental, implementadas en algunos países africanos y asiáticos, y abogadas en otros países industrializados occidentales.

El modelo de reforma estructural fue diverso en los países latinoamericanos. Bolivia, Chile, El Salvador, México y República Dominicana siguieron un modelo "sustitutivo" que cerró el sistema público y lo remplazó por el sistema privado; Argentina, Costa Rica, Panamá y Uruguay adoptaron un modelo "mixto" de dos pilares combinando una pensión abonada por el pilar público y otra por el pilar privado; y Colombia y Perú aplicaron un modelo "paralelo" en que los sistemas público y privado compiten entre sí.

La mayoría de las reformas estructurales latinoamericanas tuvo éxito en fortalecer la relación entre la cotización y el nivel de la pensión (equivalencia), mejorar la eficiencia en la gestión de las cuentas individuales y periódicamente informar su balance al asegurado, acortar el procesamiento de las prestaciones, y lograr una acumulación de capital sustancial en los fondos de pensiones así como retornos de capital entre razonables y altos.

1 Una reforma estructural cierra total o parcialmente un sistema de pensiones público mientras que una reforma paramétrica preserva el sistema público con medidas para hacerlo financieramente viable en el largo plazo.

2 En América Latina: Chile 1981, Perú 1993, Argentina y Colombia 1994, Uruguay 1996, Bolivia y México 1997, El Salvador 1998, Nicaragua 2000, Costa Rica y Ecuador 2001, República Dominicana 2003, y Panamá 2008. En Europa Central y Oriental: Hungría 1998, Polonia 1999, Letonia 2001, Bulgaria, Croacia y Estonia 2002, Lituania 2004, Eslovaquia, 2005, Macedonia 2006, y Rumanía 2008. En América Latina diez países tienen ahora sistemas públicos: Argentina, Bolivia, Brasil, Cuba, Ecuador, Guatemala, Honduras, Nicaragua, Paraguay y Venezuela (las reformas estructurales de Ecuador y Nicaragua fueron declaradas inconstitucionales o anuladas). En Europa Central y Oriental solo Eslovenia y la República Checa tienen sistemas públicos puros pero la última planea introducir un pilar privado (Müller, 2003; Mesa-Lago, 2008a; Fultz, 2012). 
Por otra parte, las reformas estructurales adolecían de importantes defectos de diseño e implementación: 1) la premisa de un paradigma aplicable a todos los países, de manera independiente de sus diferencias económico-sociales-políticas, funcionó en algunos y no en otros; 2) el sistema privado se dirigía a un mercado laboral urbano formal, pero en muchos países de América Latina predominaba el sector informal rural, por lo que la mayoría de la fuerza laboral fue excluida; 3 ) las reformas presupusieron efectos que en gran medida no ocurrieron: la cobertura se estancó o cayó, la competencia no funcionó de forma adecuada en la gran mayoría de los países (especialmente en los pequeños), los gastos administrativos eran altos, la cartera de inversión se mantuvo usualmente concentrada (debido a la falta de un mercado de capitales o a uno incipiente), la equidad de género se erosionó (los sistemas privados normalmente carecen de solidaridad social); 4) la mayoría de las reformas descuidó el pilar cero para proporcionar pensiones no contributivas o de asistencia social a los pobres; 5) el supuesto de que la propiedad de las cuentas individuales y la administración privada impedirían la intrusión del Estado y la política probó ser falsa en Argentina donde el gobierno presionó a las administradoras de pensiones a invertir en deuda pública provocando una pérdida del fondo en la crisis interna de 2001-2002 —eventualmente el Estado nacionalizó los fondos privados en Argentina y Bolivia-; 6) la supresión o reducción sustancial de las cotizaciones al sistema público generó costos fiscales de transición más altos y largos que lo previsto, aunque se sostiene que dicho costo fiscal a largo plazo hubiese sido mayor sin la reforma; y 7) la crisis financiera global de 2007-2009 redujo considerablemente el capital acumulado y su retorno en los sistemas privados, dando pie a la crítica y deteniendo las reformas estructurales - si bien hubo una recuperación posterior, algunos países usaron dichas pérdidas para justificar la re-reforma (Mesa-Lago, 2008a, 2010).

Fracasos iniciales en el diseño de los sistemas privados y el desempeño posterior a su implementación llevaron a correcciones (reformas parciales), por ejemplo, en Argentina (2003-2007), Bolivia (2007-2008) y Hungría (2000-2009), pero ellas no resolvieron los problemas fundamentales que fueron agravados por la crisis financiera global. En 2008, dos países de América Latina implementaron re-reformas de sus sistemas privados con diversos enfoques: Chile mantuvo pero mejoró dicho sistema mientras que Argentina lo cerró y transfirió todos sus fondos y asegurados al sistema público. En 2010 Bolivia y Hungría siguieron el camino argentino y nacionalizaron el pilar privado (Mesa-Lago, 2012).

\section{El caso de Chile}

Se ha escogido Chile como caso de estudio porque dicho país fue el pionero mundial tanto en la reforma estructural como en la re-reforma de pensiones. Este artículo primero analiza las características de la reforma estructural y sus efectos, después hace otro 
tanto con la re-reforma y, por último, identifica las ventajas, problemas y retos pendientes. El análisis de los efectos en el bienestar social se hace basado en el impacto de la reforma estructural y la re-reforma en diez principios básicos de la seguridad social emanados de convenciones y recomendaciones de la OIT y sistematizados por el autor (Mesa-Lago, 2008): 1) diálogo social para aprobar la reforma, 2) cobertura universal de la población, 3) trato uniforme de los asegurados, 4) solidaridad social, 5) equidad de género, 6) suficiencia en las prestaciones, 7) eficiencia y costo administrativo razonable, 8) participación social en la gestión del sistema, 9) rol del Estado y supervisión, y 10) sostenibilidad financiera.

La reforma estructural del sistema público de pensiones en Chile se hizo por el LeyDecreto 3.500 de 11 abril de 1980 y entró en vigor en 1981. La reforma cerró múltiples regímenes separados de pensiones y los remplazó con un sistema privado. A los que estaban asegurados se le dio un plazo para que decidieran si quedarse en el sistema público o cambiarse al privado, mientras que todos los nuevos ingresantes en la fuerza laboral fueron obligados a afiliarse al sistema privado. En 2011, después de 30 años de la reforma estructural, $98,5 \%$ de todos los cotizantes estaba en el sistema privado y solo $1,5 \%$ en el sistema público, pero las proporciones respectivas de pensionados eran 55,5\% y 45,5\% (SP, 2012). Dicha reforma trajo algunas mejoras pero varios de sus presuntos efectos positivos no se materializaron y el sistema privado adolecía de serias fallas. Tomó 28 años para aprobar una re-reforma que mejorase el sistema, por la Ley 20.255 de 17 marzo de 2008 (Mesa-Lago, 2008b, 2012).

\subsection{Características y efectos de la reforma estructural y el sistema privado}

\subsubsection{Diálogo social}

La OIT recomienda el diálogo social con los sectores involucrados previo a cualquier tipo de reforma de pensiones, pero la gran mayoría de las reformas estructurales que privatizaron las pensiones en la mitad de América Latina se decidieron sin dicho diálogo, lo que más tarde condujo a problemas de legitimidad. El gobierno militar autoritario de Chile promulgó la ley de reforma con un Congreso totalmente controlado, sindicatos prohibidos o sometidos y sin discusión pública. Los reformadores sostuvieron que el sistema público estaba en quiebra, era ineficiente y financieramente insostenible, mientras que el sistema privado mejoraría la cobertura, eficiencia y prestaciones, reduciría los costos administrativos y sería financieramente sostenible en el largo plazo.

\subsubsection{Cobertura universal}

La cobertura de la población económicamente activa (PEA) en el sistema privado cayó de $73 \%$ en 1973 al 64\% en 1980 (el año en que se promulgó la reforma) y al 29\% en 
1982. Aunque la cobertura creció a 61,2\% en 2007 (el año antes de la re-reforma) era todavía inferior a los niveles de 1973 y 1980. La cobertura aumentó a 62,8\% en 2008 y disminuyó y se estancó en 2009-2010 debido a la crisis global. Datos de encuestas que incluyen a las fuerzas armadas y a la policía muestran mayor cobertura pero prácticamente estancada: $62,2 \%$ en 1990 y $62,9 \%$ en 2006 . Los afiliados que cotizan regularmente mermaron de $73,6 \%$ en 1990 a $54,6 \%$ en 2008 . La cobertura de la población de 65 y más años por todas las pensiones declinó de 73\% al 60,7\% en 1990-2006 (Cuadro 1). En 2008, los trabajadores autónomos (por cuenta propia) eran el 17,7\% de la PEA; su afiliación al sistema de pensiones era voluntaria y representaba sólo el 3,5\% del total de cotizantes; los trabajadores familiares no remunerados estaban legalmente excluidos. La cobertura aumentaba con el ingreso, la educación, la ubicación urbana versus la rural y en el sector público versus en el privado (Rofman y Oliveri, 2012).

\subsubsection{Trato uniforme}

La reforma unificó 33 de los 35 regímenes de pensiones separados que existían en 1979 (con diferencias significativas entre ellos), normalizó sus condiciones de elegibilidad eliminando algunas importantes desigualdades, aumentó sus edades de jubilación y sus cotizaciones, y unificó su gestión en el Instituto de Normalización Previsional (INP). Aunque el gobierno militar ejecutó la reforma y proclamó su excelencia sobre el sistema público, las fuerzas armadas y la policía fueron excluidas del sistema privado y mantienen sus regímenes previos de prestación definida con condiciones de derecho y pensiones más generosas, en buena parte financiadas por el Estado. En 2006, 1,3\% del PIB se gastó en cubrir el déficit del régimen de las fuerzas armadas, mientras que sólo el $0,4 \%$ fue asignado a las pensiones no contributivas y $0,1 \%$ a las pensiones mínimas.

\subsubsection{Solidaridad social}

Los sistemas privados normalmente carecen de solidaridad social endógena porque la cuenta individual pertenece al trabajador asegurado y no se comparte con otros. La poca solidaridad social que existía en Chile estaba fuera del sistema privado, a través de la financiación estatal de las pensiones no contributivas, la pensión mínima garantizada y los programas de protección social. 
Cuadro 1: Cobertura por Pensiones de la PEA y la Población de 65 y más años, y Afiliados que Contribuyen Activamente, 1973-2011 (en porcentajes)

\begin{tabular}{|c|c|c|c|c|}
\hline \multirow{2}{*}{ Años* } & \multicolumn{2}{|c|}{$\begin{array}{c}\text { Cobertura de la PEA } \\
\text { basada en: }\end{array}$} & \multirow{2}{*}{$\begin{array}{l}\text { Afiliados que } \\
\text { cotizan }^{* * *}\end{array}$} & \multirow{2}{*}{$\begin{array}{l}\text { Cobertura } \\
\text { población } \\
\text { edad } 65+\end{array}$} \\
\hline & Estadísticas $^{* *}$ & Encuestas & & \\
\hline 1973 & 73,0 & & & \\
\hline 1980 & 64,0 & & & \\
\hline 1982 & 29,0 & & 73,6 & \\
\hline 1990 & 46,8 & 62,2 & 61,2 & 73,0 \\
\hline 1992 & 51,8 & 61,1 & 60,7 & 66,5 \\
\hline 1994 & 51,8 & 62,3 & 57,2 & 68,2 \\
\hline 1996 & 55,7 & 62,4 & 55,7 & 65,9 \\
\hline 1998 & 53,4 & 58,9 & 52,8 & 62,2 \\
\hline 2000 & 52,9 & 58,0 & 50,9 & 63,7 \\
\hline 2003 & 55,8 & 58,7 & 51,8 & 63,9 \\
\hline 2006 & 58,0 & 62,9 & 51,5 & 60,7 \\
\hline 2007 & 61,2 & & 53,8 & \\
\hline 2008 & 62,8 & & 54,6 & \\
\hline 2009 & 60,3 & 73,1 & 51,7 & 57,2 \\
\hline 2010 & 60,3 & & 54,5 & \\
\hline 2011 & 61,8 & & & \\
\hline
\end{tabular}

Notas:

${ }^{*}$ Diciembre de cada año.

${ }^{* *}$ Desde 1982 basada en afiliados que cotizaron en el último mes.

**** Porcentaje de afiliados que cotizaron en el último mes.

Fuente: Estadísticas de SAFP, 2009, actualizadas con AIOS, 2010-2011 y Arenas de Mesa, 2012; encuestas y cobertura 65+ de Rofman, Locchetti y Ourens, 2008, y Rofman y Oliveri, 2012; afiliados que cotizan son cálculos del autor basados en SAFP, 2009 y AIOS, 2010-2011. 


\subsubsection{Equidad de género}

Los sistemas públicos atenúan las desigualdades de género; casi ninguno en América Latina ha aumentado el número de años de cotización para obtener la pensión mínima, hay transferencias de hombres a mujeres, y utilizan tablas unisexo para calcular las pensiones. Por el contrario, los sistemas privados acentúan las desigualdades de género, además de las creadas por el mercado de trabajo. En Chile, las desigualdades de género existían con anterioridad a la reforma estructural, como una cobertura de seguro inferior para las mujeres respecto a los hombres (debido a una menor tasa de participación femenina y una mayor proporción en el trabajo informal), pero las desigualdades se acentuaron por el sistema privado. Las mujeres representaban el 38,8\% de los cotizantes totales versus $61,2 \%$ de los hombres, y el 54\% de las pensiones recibidas por los adultos mayores versus el 69,4\% de los hombres (AIOS, 2011; Rofman y Oliveri, 2012). Las mujeres recibían pensiones inferiores a las de los hombres por causa de sus frecuentes salidas del mercado laboral para criar a sus hijos, el aumento en los años de cotización requeridos para obtener la pensión mínima, una menor densidad de cotización que la de los hombres (42\% and 61\% respectivamente), y un cálculo de la pensión basado en el fondo acumulado en la cuenta individual y tablas de mortalidad diferenciadas por sexo. Teniendo un fondo capitalizado menor y una superior esperanza de vida, la renta vitalicia de las mujeres era menor que las de los hombres, y sus tasas de remplazo respectivas eran 35\% y 46\%. Una prima igual de discapacidad-sobrevivientes se cargaba a ambos sexos, aunque la mujer sufría menor riesgo y, por tanto, pagaba más que el hombre. Por otra parte, los hombres no tenían derecho a una pensión de sobreviviente cuando morían sus esposas aseguradas.

\subsubsection{Suficiencia en las prestaciones}

La reforma estructural y el sistema privado fortalecieron la relación entre la cotización y el nivel de la pensión contributiva (equivalencia). Antes de dicha reforma había una pensión no contributiva concedida a los pobres sometida a prueba de ingreso (PASIS), que continuó pero limitada por un tope en el número de beneficiarios, así como listas de espera y la disponibilidad de recursos fiscales. La mayoría de los regímenes públicos previos concedía una pensión mínima con un número relativamente pequeño de años de cotización, elevados a 20 por la reforma estructural. Bajo ésta, el Estado garantizaba una pensión mínima a aquellos en el sistema privado que tenían 20 años de cotización y cuya suma en la cuenta individual era insuficiente para financiar esa pensión (el Estado pagaba la diferencia). La pensión mínima era insuficiente, ya que promediaba $62 \%$ del salario mínimo en 2007 y mostraba una tendencia decreciente. Se proyectaba que sólo la mitad de los asegurados obtendría la pensión mínima: el 35\% de los hombres y el $60 \%$ de las mujeres. Parte de los asegurados al tiempo de la jubilación carecía del derecho a recibir una pensión mínima (por faltarle los años de cotización requeridos) o 
a una pensión no contributiva (por no cumplir con la prueba de ingreso o por falta de recursos fiscales). Las pensiones contributivas eran automáticamente indexadas a una unidad monetaria (Unidad de Fomento: UF).

\subsubsection{Eficiencia y costos administrativos razonables}

La unificación en el INP de los numerosos regímenes separados de pensiones que existían (excepto las fuerzas armadas y la policía), resultó en una reducción de costos en el sistema público. El sistema privado era (es) administrado por empresas privadas ad hoc con fines de lucro: Administradoras de Fondos de Pensiones (AFP). Estas cobran una comisión como un porcentaje del salario imponible para la administración del programa de vejez, así como una comisión fija que tenía efectos regresivos; además una prima para cubrir los riesgos de discapacidad-sobrevivientes se cobra por las AFP y se transfiere a compañías de seguros comerciales. La competencia no funcionó en la realidad tan bien como se esperaba: el número de AFP cayó de 21 a 5 en 1994-2008, la concentración de cotizantes en las tres mayores AFP creció de $67,1 \%$ a $86,4 \%$, y se impusieron restricciones a la libertad de elección para cambiar de AFP. A pesar de la presunción de que disminuirían los costos administrativos debido a la competencia y la administración privada, la comisión total promedio sobre el salario subió de 2,44\% a $2,68 \%$ entre 1981 y 2008 (Cuadro 2).

Cuadro 2: Costos Administrativos en el Sistema Privado, 1981 y 2007-2012

\begin{tabular}{|c|c|c|c|c|}
\hline \multirow[b]{2}{*}{ Años } & \multicolumn{3}{|c|}{ Costo (\% del salario imponible) } & \multirow{2}{*}{$\begin{array}{l}\text { Costo total/ } \\
\text { descuento para } \\
\text { depósito }(\%)^{\mathrm{c}}\end{array}$} \\
\hline & Vejez $^{a}$ & $\mathrm{D} \& \mathrm{~S}^{\mathrm{b}}$ & Total & \\
\hline 1981 & - & - & 2,44 & 24,4 \\
\hline 2007 & 1,71 & 0,73 & 2,40 & 24,4 \\
\hline 2008 & 1,74 & 0,94 & 2,68 & 26,8 \\
\hline 2009 & 1,55 & $1,87^{\mathrm{d}}$ & 3,38 & 33,8 \\
\hline 2010 & 1,49 & $1,49^{d}$ & 2,99 & 29,9 \\
\hline 2012 & 1,43 & $1,26^{\mathrm{d}}$ & 2,69 & 26,9 \\
\hline
\end{tabular}

Notas:

* Comisión neta pagada a la AFP.

** Prima de discapacidad y sobrevivientes a compañías de seguro.

**** El depósito es $10 \%$ del salario imponible. d En 2009, una prima única para hombres y mujeres aumentó la tasa pero esta bajó en 2010-2012.

Fuente: Mesa-Lago, (2013); 2009-2010 basado en CUSP, 2011; 2012 de Arenas de Mesa, (2012). 


\subsubsection{Participación social en la gestión}

Antes de la reforma estructural, la mayoría de los fondos de pensiones tenía participación tripartita en su administración a través de representantes de los trabajadores, empleadores y el gobierno, aunque había diferencias en cuanto a su efectividad y forma de elección de los representantes. La reforma eliminó la participación social en la gestión de las AFP y la Superintendencia de las AFP, a pesar de que los trabajadores son propietarios de sus fondos de pensiones.

\subsubsection{Rol del Estado y supervisión}

Bajo la privatización, en teoría, el Estado jugaría un "rol subsidiario" al mercado pero, en la práctica, este tiene un papel clave incluso en Chile donde virtualmente todos los asegurados están en el sistema privado: la afiliación y las cotizaciones son obligatorias para los empleados asalariados; el gobierno regula, supervisa y garantiza el sistema; y financia el costo fiscal de la reforma durante la transición (ver 1.10). Prevaleció la atomización debido a múltiples regulaciones y entidades supervisoras: la Superintendencia de las AFP, una agencia técnica relativamente autónoma, para el sistema privado; la Superintendencia de Seguridad Social y el INP para el resto del sistema público. Los regímenes de las fuerzas armadas y la policía no están sometidos a la supervisión estatal, salvo por la auditoría general.

\subsubsection{Sostenibilidad financiera}

La reforma estructural suprimió la cotización del empleador y la transfirió al trabajador, que paga el 10\% sobre el salario/ingreso imponible, para el depósito en la cuenta individual, más todas las comisiones, lo cual viola la norma mínima de la OIT que estipula que la cotización del trabajador no debe exceder el 50\% de la cotización total. La acumulación de capital en todas las cuentas individuales aumentó de 3,9\% a 64,4\% del PIB en 1982-2007, aunque decreció a 52,8\% en 2008 debido a la crisis global. La tasa de retorno o rentabilidad bruta real (sin descontar el costo administrativo y ajustada a la inflación) del capital total invertido fluctuó de $28,5 \%$ en 1982 a $-18.9 \%$ en 2008 ; el retorno real anual promedio desde el inicio del sistema se ha desempeñado bastante bien pero con una caída constante de $20,6 \%$ a $8,8 \%$ en el período. La composición de la cartera por instrumento de inversión se diversificó gradualmente en los 28 años de la reforma estructural: en deuda pública disminuyó de $35 \%$ a $14 \%$ y en las instituciones financieras de $33 \%$ al $30 \%$, mientras que la inversión en acciones aumentó del $12 \%$ al $14 \%$ y en las emisiones extranjeras del $13 \%$ al $28 \%$; la mitad de la cartera total estaba concentrada en los dos últimos instrumentos en 2007, lo cual provocó una fuerte caída en la tasa de retorno en 2008 por la crisis financiera global (Cuadro 3). Antes de esta se introdujeron "multifondos" en Chile (más tarde en Colombia, México y Perú), donde 
el asegurado puede elegir entre cinco carteras de diverso riesgo. Según el asegurado se acerca a la edad de jubilación, debería moverse hacia una cartera más conservadora y de hecho, cuando estalló la crisis, el $80 \%$ de ellos tenía su fondo invertido en las dos carteras menos riesgosas. Por el contrario, quienes en medio de la crisis cambiaron carteras desde alto a bajo riesgo, perdieron una suma importante de sus fondos y no habían recuperado su valor en 2010 (CUSP, 2011).

Cuadro 3: Capital Acumulado en el Fondo, Retorno Real del Capital, Composición de la Cartera por Instrumentos Principales y Costo Fiscal de la Transición en el Sistema Privado de Pensiones, 1982-2011

\begin{tabular}{|c|c|c|c|c|c|c|c|c|c|}
\hline \multirow{2}{*}{ Años ${ }^{*}$} & \multicolumn{2}{|c|}{$\begin{array}{c}\text { Capital } \\
\text { acumulado }\end{array}$} & \multicolumn{2}{|c|}{ Retorno real (\%) } & \multicolumn{4}{|c|}{ Distribución de la cartera por instrumentos (\%) } & \multirow{2}{*}{$\begin{array}{c}\text { Costo } \\
\text { fiscal } \\
(\% \\
\text { PIB) }\end{array}$} \\
\hline & $\begin{array}{c}\text { Millones } \\
\text { US\$ }\end{array}$ & $\begin{array}{c}\% \\
\text { PIB }\end{array}$ & $\begin{array}{l}\text { Desde } \\
\text { inicio }^{* *}\end{array}$ & $\begin{array}{l}\text { Último } \\
\text { año }\end{array}$ & $\begin{array}{l}\text { Deuda } \\
\text { pública }\end{array}$ & $\begin{array}{c}\text { Instituciones } \\
\text { financieras }\end{array}$ & $\begin{array}{c}\text { Acciones } \\
\text { nacionales }\end{array}$ & $\begin{array}{l}\text { Emisiones } \\
\text { extranjeras }\end{array}$ & \\
\hline 1982 & 600 & 3,9 & 20,6 & 28,5 & - & - & - & - & $-6,4$ \\
\hline 1984 & 1.200 & 7,7 & 16,6 & 3,6 & - & - & - & - & $-7,6$ \\
\hline 1999 & 34.501 & 5,3 & 11,3 & 16,3 & 34,6 & 33,2 & 12,4 & 13,4 & $-5,9$ \\
\hline 2000 & 35.886 & 59,8 & 10,9 & 4,4 & 35,7 & 35,1 & 11,6 & 10,9 & $-6,0$ \\
\hline 2001 & 35.461 & 55,0 & 10,7 & 6,7 & 35,0 & 32,5 & 10,6 & 13,1 & $-6,0$ \\
\hline 2002 & 35.515 & 55,8 & 10,3 & 3,0 & 30,0 & 34,2 & 9,9 & 16,2 & $-5,9$ \\
\hline 2003 & 47.690 & 64,5 & 10,3 & 10,6 & 24,7 & 26,3 & 14,5 & 23,7 & $-5,8$ \\
\hline 2004 & 60.799 & 59,1 & 10,2 & 8,9 & 18,7 & 28,5 & 15,7 & 26,8 & $-5,5$ \\
\hline 2005 & 74.750 & 59,4 & 10,0 & 4,6 & 16,4 & 28,9 & 14,7 & 30,2 & $-5,2$ \\
\hline 2006 & 88.632 & 61,0 & 10,2 & 15,8 & 13,1 & 26,3 & 17,0 & 32,0 & $-5,1$ \\
\hline 2007 & 111.037 & 64,4 & 10,0 & 5,0 & 7,8 & 30,3 & 14,5 & 35,6 & $-5,0$ \\
\hline 2008 & 74.313 & 52,8 & 8,8 & $-18,9$ & 14,3 & 29,7 & 13,8 & 28,5 & $-4,9$ \\
\hline 2009 & 118.053 & 65,3 & 9,2 & 22,5 & 9,7 & 18,9 & 13,9 & 43,9 & $-4,8$ \\
\hline 2010 & 124.726 & 61,9 & 9,3 & 12,0 & 10,0 & 16,6 & 14,6 & 45,0 & $-4,7$ \\
\hline 2011 & 153.009 & 64,0 & 9,1 & 7,0 & 14,5 & 16,2 & 26,1 & 43,2 & \\
\hline
\end{tabular}

Notas:

"Diciembre, salvo junio 2010 y 2011.

"* Promedio anual desde el inicio del sistema hasta el final de cada año.

Fuente: AIOS, 1999-2011; FIAP, 2011; última columna de Mesa-Lago, 2013. 
El Estado financia todos los costos de la transición: el déficit operacional resultante del cierre del sistema público, el valor de las cotizaciones pagadas al sistema público por los asegurados que se cambiaron al sistema privado ("bono de reconocimiento"), el costo diferencial de las pensiones mínimas y las pensiones no contributivas; también garantiza las pensiones en caso de quiebra de una AFP. Contrariamente a las proyecciones iniciales, los costos de transición eran alrededor de 5\% del PIB en 2008 a pesar de los 28 años transcurridos desde la reforma estructural y una reducción en el déficit operacional (Cuadro 3). Esos costos son financiados por toda la población a través de impuestos (sobre todo al consumo), incluyendo a los pobres y otros no cubiertos, con efectos regresivos. A diferencia de otras reformas de América Latina, la chilena pudo financiar los altos costos de la transición porque generó un superávit fiscal constante (CPS, 2011).

\subsection{Características y efectos de la re-reforma de pensiones ${ }^{3}$}

Las AFP con enorme poder, derivado de administrar fondos de pensiones por valor de $64 \%$ del PIB, lograron obstruir la necesaria re-reforma durante 28 años a pesar de crecientes críticas bajo sucesivos gobiernos democráticos desde 1990 respecto a las fallas del sistema privado. A continuación se describen las características de la re-reforma y se evalúan sus efectos. Algunos de estos fueron medidos por dos encuestas tomadas en 2009, por lo tanto, sólo durante el primer año de la re-reforma; una encuesta realizada en 2011, cuyos resultados no estaban disponibles al terminar este trabajo, juzgará los resultados en 2010-2011.

\subsubsection{Diálogo social}

En 2006, la Presidente Michelle Bachelet nombró un consejo asesor con representación de todos los sectores de la sociedad para: estudiar la re-reforma, discutirla en numerosas reuniones públicas — una de ellas con expertos internacionales - y preparar un informe proponiendo cambios para corregir las debilidades del sistema privado; el 90\% de las recomendaciones del consejo se incorporaron en el proyecto legal presentado al Parlamento en 2007, que fue aprobado como Ley 20.255 y entró en vigor en julio de 2008 (Gobierno de Chile, 2008; Arenas de Mesa, 2010).

\subsubsection{Cobertura universal}

La ley de re-reforma ordenó la cobertura obligatoria gradual de los trabajadores autónomos a partir de 2012 (precedida por un proceso de información y educación); inicial-

\footnotetext{
${ }^{3}$ Parte de esta sección se basa en Mesa-Lago, 2008b, 2012, 2013.
} 
mente cotizaron $10 \%$ del $40 \%$ de su ingreso imponible anual, aumentándose la última proporción de forma paulatina hasta que en 2015 cotizarán sobre el total de sus ingresos gravables. Como incentivo para la afiliación, los trabajadores autónomos son ahora elegibles para las asignaciones familiares, la protección contra riesgos de empleo y los aportes fiscales solidarios. Los autónomos registrados subieron $24 \%$ en $2009-2010$, pero todavía eran sólo el 3,5\% del total de los afiliados, el mismo porcentaje que en 2008. Se concede afiliación voluntaria a los trabajadores no remunerados (incluyendo amas de casa) y se otorga un subsidio estatal a los trabajadores jóvenes de bajo ingreso durante sus primeros dos años de afiliación. Sin embargo, el número de afiliados voluntarios en 2010 era todavía pequeño $(0,5 \%$ del total) y también había pocos jóvenes inscritos como resultado de la re-reforma (CUSP, 2011). El número de cotizantes al sistema privado aumentó $15,7 \%$ en $2007-2011$, de 4,3 a 5 millones; como porcentaje de la PEA, sin embargo, hubo una disminución de $62,8 \%$ en 2008 a 60,3\% en 2009, debido al efecto de la crisis global, pero con la recuperación económica subió a $61,8 \%$ en 2011. La cobertura de la PEA por todas las pensiones se expandió de $62,9 \%$ a 73,1\% en 2006-2009 (Cuadro 1). La cobertura contributiva y no contributiva de los adultos mayores saltó de $23,9 \%$ en 2008 a 51,1\% en 2009 y se proyecta alcanzará $97 \%$ en 2025 (Arenas de Mesa y otros, 2008; Arenas de Mesa, 2010). La re-reforma creó una pensión básica universal no contributiva para ampliar la cobertura de la población pobre y de bajos ingresos (véase 2.4).

\subsubsection{Trato uniforme}

La re-reforma no ha podido corregir la desigualdad en el trato ya que mantiene excluidos a los regímenes de las fuerzas armadas y de la policía, que reciben prestaciones superiores a las del sistema general y con fuertes subvenciones fiscales. A pesar de algunos intentos legales fallidos y la presión sobre las fuerzas armadas, estas permanecían fuera del sistema privado a fines de 2013.

\subsubsection{Solidaridad social}

La re-reforma creó dos nuevas "pensiones solidarias" financiadas por el Fisco: a) La Pensión Básica Solidaria (PBS) de vejez e invalidez, para sustituir gradualmente a la PASIS, poner fin a las listas de espera y restricciones fiscales que impedían su universalidad (la PBS se otorga a los afiliados que anteriormente no tenían derecho a una pensión mínima o a la PASIS). A partir de 2008, el PBS se concedió al $40 \%$ de la población de menores ingresos, que no recibe otra pensión, tiene 65 años o más y es residente por 20 años; y gradualmente se extendió al $60 \%$ de la población de menores ingresos en 2011. b) El Aporte Previsional Solidario (APS) que remplaza gradualmente a la pensión mínima garantizada y es siempre mayor que la PBS. El APS complementa la pensión contributiva de los que tengan 65 años de edad o más y cuyo ingreso es 
bajo; el APS disminuye según el monto de la pensión contributiva y termina cuando esta supera un techo que se aumentó gradualmente hasta 2012, por lo que tiene efectos progresivos. Las condiciones de elegibilidad para el APS son similares a las de la PBS. Las cotizaciones de los trabajadores jóvenes son subsidiadas durante sus dos primeros años de afiliación.

\subsubsection{Equidad de género}

Para hacer frente a las desigualdades de género, la re-reforma introdujo varias medidas. Las madres afiliadas al sistema privado o beneficiarias de una pensión de PBS o de sobreviviente, independientemente de sus condiciones socio-económicas, reciben un bono de maternidad equivalente al 10\% de 18 salarios mínimos por cada hijo nacido vivo; el bono es acreditado a la cuenta individual de la madre cuando nace el hijo, a partir de entonces devenga interés anual y se hace efectivo a los 65 años, aumentando el nivel de la pensión. En 2009-2010, 475.548 mujeres habían recibido el bono. El seguro de discapacidad-sobrevivencia carga una prima igual (pagada por los empleadores desde el 2011) a hombres y mujeres, pero como estas tienen menor incidencia de riesgo y utilizan menos dicho seguro, la prima excedente pagada por las mujeres ahora se acredita a sus cuentas individuales (0,3\% de la prima en 2010). No obstante, persisten las tablas de mortalidad diferenciadas por género. En caso de divorcio, un juez puede ordenar el traslado de los fondos acumulados en la cuenta individual durante el matrimonio, desde uno de los cónyuges al otro (generalmente la mujer), hasta un máximo del $50 \%$. Las amas de casa pueden tener afiliación voluntaria y recibir cotizaciones de sus cónyuges o de sus propios ingresos. Un programa de ampliación de guarderías infantiles facilita el empleo de la mujer, mejora el ingreso familiar y aumenta su afiliación al sistema de pensiones. Los cónyuges masculinos ahora tienen derecho a una pensión de sobreviviente (CUSP, 2011; Arenas de Mesa, 2012; SP, 2012).

Ha mejorado ligeramente la distribución porcentual de la cobertura basada en cotizantes por género pero todavía favorece a los hombres: la proporción femenina del total fue de $38,8 \%$ en 2008 y $39,4 \%$ en 2011 . Por el contrario, la distribución de las pensiones solidarias (PBS y APS) es muy favorable a las mujeres: 62,8\% versus el 37,2\% de los hombres en 2012, y la pensión solidaria promedio femenina era $4 \%$ superior a la masculina (SAFP, 2009; SP, 2012).

\subsubsection{Suficiencia de las prestaciones}

El valor de la PBS en 2008-2009 fue 50\% mayor que el de la anterior PASIS, después se ajusta anualmente al índice de precios al consumidor (IPC). La PBS aumentó los ingresos a un promedio general de $34 \%$ en 2009: $72 \%$ a los que están en el 5\% del estrato inferior y $57 \%$ a aquellos en el $10 \%$ más bajo. El APS debería mejorar las pensiones contributivas, proporcionar incentivos para la afiliación y la cotización, y reducir los 
incentivos para la evasión; desde 2010 también se ajusta al IPC. La distribución de los gastos por pensiones solidarias en 2010 fue: $89 \%$ a la PBS y $11 \%$ al APS. El número de beneficiarios en ambos programas casi se duplicó desde 603.427 en 2008 a 1,1 millones en 2012 (39,5\% de todos los beneficiarios en el país) y se proyecta que llegará a 1,9 millones en 2025. La pobreza se redujo un 2,7\% entre los que tienen 65 años de edad o más y un $0,7 \%$ entre la población total. En 2010 se implementó un nuevo mecanismo de focalización que mide la pobreza y obtiene datos del ingreso familiar: la PBS se otorgó a $84,5 \%$ de las personas que están en el $60 \%$ de ingreso más pobre en la población, pero también fue impropiamente pagado al $8 \%$ en el $30 \%$ de mayor ingreso, lo cual obligó a una modificación y suspensión de beneficios al $0,2 \%$ del total de beneficiarios en 2010 (CPS, 2011). La re-reforma creó un programa de ahorro voluntario colectivo con cotizaciones negociadas por los empleadores y asegurados (también puede ser solo por los empleadores), con beneficios tributarios, tales como el pago de impuestos diferidos hasta que los fondos son retirados. Sin embargo, en 2010 muy pocos empresarios participaban en este programa (CUSP, 2011; SP, 2012). Como antes, las pensiones contributivas se indexan automáticamente a la unidad monetaria (UF). Desde 2012 y, particularmente en 2013, ha habido críticas de los sindicatos sobre el todavía bajo nivel de la pensión contributiva (ver 4).

\subsubsection{Eficiencia y costos administrativos razonables}

La re-reforma estableció las siguientes medidas para estimular la competencia y reducir los costos administrativos: a) licitación cada dos años a fin de que la AFP que ofrece la comisión más baja gane la afiliación de quienes ingresan anualmente al mercado laboral, los recién inscritos deben permanecer en la AFP seleccionada por dos años y más tarde pueden cambiar a otra que cobre una comisión neta inferior (la comisión reducida también se aplica a los afiliados que ya estaban en la AFP ganadora); b) eliminación de la comisión de suma fija que tenía efectos regresivos; c) autorización a los bancos para administrar las cuentas individuales en competencia con las AFP; y d) substitución de la licitación individual por cada AFP para escoger las compañías de seguros comerciales por una licitación colectiva universal independientemente de la afiliación la cual debe reducir los costos. El número de AFP aumentó de cinco en 2008 a seis en 2011 y la concentración disminuyó ligeramente. En 2012, la comisión por vejez cargada por la AFP que ganó la licitación era $1,14 \%$ comparada con la comisión más alta de 2,36\%. La comisión total promedio aumentó de 2,68\% a 3,38\% en 2008-2009 porque la abolida comisión fija se excluía en los promedios anteriores y ahora se agrega como costo variable, además que se expandió la cobertura de los riesgos de discapacidad-sobrevivencia. Sin embargo, como resultado de la licitación, la comisión total declinó a 2,69\% en 2012, al mismo nivel que en 2008. Los beneficiarios de la PBS y los pensionados de ingreso medio fueron eximidos en 2011 de la cotización al programa de salud (Cuadro 2; CUSP, 2011; ISSA, 2011-2012; SP, 2012). 


\subsubsection{Participación social en la gestión}

Para compensar la falta de participación de los trabajadores y pensionados en la gestión del sistema privado, la re-reforma creó una comisión de usuarios, compuesta por cinco representantes, uno de cada: trabajadores, pensionados, asegurados en el sistema público, académicos y AFP. Los representantes expresan sus opiniones sobre el desempeño del sistema, monitorean el cumplimiento de los objetivos de la re-reforma y desarrollan estrategias para la educación de los afiliados; la comisión publicó informes evaluativos en 2010 y 2011. También se creó un Fondo de Educación Previsional, financiado por el Estado y las AFP, para difundir información y educar al público en materia de pensiones, así como centros y asesores privados que responden consultas del público y ayudan a los asegurados a reclamar prestaciones y seleccionar AFP, multifondos y opciones de pensión. A pesar de estas medidas positivas, persiste la falta de conocimiento adecuado del sistema de pensiones; en 2010 había 536 asesores privados que procesaban el 22\% de las consultas, pero el asegurado necesita mejor información sobre su ubicación y servicios (CUSP, 2011).

\subsubsection{Rol del Estado y supervisión}

Bajo la re-reforma, el papel del Estado se ha ampliado considerablemente en: otorgar prestaciones, infundir solidaridad social y equidad de género, promover la competencia, consolidar la supervisión y garantizar la solidez financiera de las prestaciones futuras. La Superintendencia de las AFP fue abolida y sustituida por una Superintendencia de Pensiones unificada, que supervisa los sistemas de pensiones privados y públicos (excluyendo la fuerza armada y la policía), así como las nuevas pensiones solidarias (se eliminó la administración de pensiones mínimas por las AFP), los centros que proveen las pensiones y otros organismos públicos relacionados. El INP se transformó en el Instituto Nacional de Previsión; el tiempo de espera en sus centros se redujo de una hora a 15 minutos, el índice de satisfacción general fue de $89 \%$ y el de servicio adecuado fue $96 \%$; una nueva "ventanilla única" atiende a todos los tipos de pensiones por lo que ha agilizado el servicio (CUSP, 2011). Se ampliaron los poderes de la Subsecretaría de Previsión Social para diseñar políticas sociales públicas y el seguimiento de la re-reforma (Arenas de Mesa, 2012).

\subsubsection{Sostenibilidad financiera}

Debido principalmente a la recuperación económica, así como a la mejora del sistema privado, el valor total del fondo se duplicó en 2008-2011: de US\$ 74.313 millones a US\$153.009 millones, un récord histórico. El retorno real promedio anual del capital desde el inicio del sistema creció del 8,8\% al 9,1\%, y el promedio en los últimos 12 meses mejoró de $-18.9 \%$ en 2008 a 22,5\% en 2009 pero menguó a 7\% en 2011 (Cuadro 
3). En 2010, la distribución de los multifondos por cartera de riesgo parecía estar mejor equilibrada que en 2008 teniendo en cuenta la edad del asegurado: $53 \%$ estaba en las dos carteras menos riesgosas, $37 \%$ en la intermedia y $10 \%$ en las dos más riesgosas. Desde 2011, la prima de discapacidad y sobrevivientes se transfirió del asegurado al empleador, reduciendo la carga del primero pero sin cumplir aún la norma de la OIT de que no más del $50 \%$ de la cotización total sea pagada por los trabajadores. El Estado proporciona estímulos fiscales a los empresarios para cotizar a las cuentas individuales voluntarias de sus empleados, pero con escasos resultados hasta ahora - se introdujeron modificaciones para intentar mejorar esto. Las nuevas prestaciones y organismos son financiados por impuestos generales y recursos ahorrados por la terminación de algunos pagos en el sistema público. En 2010, hubo una mejoría en la recaudación y un ligero descenso en la tendencia histórica de la mora patronal (CUSP, 2011). ${ }^{4}$ Los costos fiscales de la reforma estructural en 2010 fueron 4,7\% del PIB, mientras que los costos fiscales de la re-reforma fueron $0,68 \%$ y se proyecta un cénit de $1,3 \%$ en 2030 . El costo total de las pensiones (contributivas y solidarias) se proyecta en $2,4 \%$ del PIB para 2020, pero esta estimación no es comparable con las anteriores (CPS, 2011).

La re-reforma sentó las bases para asegurar su sostenibilidad financiera a largo plazo: a) creó un fondo de pensiones solidario con una reserva para financiar las nuevas prestaciones; b) el Ministerio de Hacienda desarrolla cada tres años un estudio actuarial del fondo y el nuevo sistema, asigna recursos fiscales según la ley de presupuesto y prepara informes anuales sobre los recursos necesarios; c) la Superintendencia de Pensiones y el Ministerio realizan informes actuariales cada cinco años, para evaluar los efectos de variables demográficas, financieras y de cambios en el comportamiento de los afiliados sobre las tasas de remplazo y las necesidades financieras; d) un consejo asesor de pensiones compuesto por expertos cualificados monitorea el impacto fiscal de la re-reforma, su sostenibilidad y posibles modificaciones requeridas; y e) la comisión de usuarios elabora informes anuales sobre la evolución de la re-reforma (CCP, 2011; CUSP, 2011). En 2010, un informe de la Universidad de Chile confirmó que el nuevo sistema estaba en equilibrio actuarial en 2009-2025 (Arenas de Mesa, 2010). El primer informe actuarial en 2010 mostró que el sistema será capaz de cumplir sus obligaciones hasta el año 2030 (CPS, 2011).

\footnotetext{
4 A mediados de 2011, la mora equivalía al 1,1\% del total acumulado en el fondo en 30 años.
} 


\section{Conclusiones: ventajas y problemas/retos de la re-reforma}

\subsection{Ventajas}

» La re-reforma fue precedida por un diálogo social amplio, y promueve la mayoría de los principios de la seguridad social, con efectos positivos en el bienestar social;

» procura una cobertura más amplia mediante la afiliación legal obligatoria gradual de los trabajadores autónomos y les da incentivos para afiliarse al sistema;

» establece estímulos para la afiliación y pago de las cotizaciones y promueve las cotizaciones voluntarias de los empleadores;

» otorga una pensión básica solidaria a todos los grupos pobres y de bajos ingresos, sin una lista de espera o restricciones fiscales;

» resuelve la falta de protección a los afiliados que antes no calificaban para una pensión mínima o no contributiva;

» mejora las pensiones existentes con un aporte fiscal calculado como un porcentaje de la pensión contributiva; dicho aporte disminuye con el monto de la pensión y se elimina cuando excede un límite máximo;

» alcanzó la meta de 1 millón de beneficiarios de pensiones solidarias antes de lo previsto;

» otorga un bono de maternidad a todas las madres, así como otras prestaciones para mitigar las desigualdades de género;

» estimula la competencia e introduce mecanismos para reducir los costos administrativos;

» crea una entidad para facilitar el proceso de reclamo de las nuevas prestaciones, informar al asegurado y promover la educación previsional;

» instituye participación social representativa a través de la comisión de usuarios que monitorea la re-reforma;

» unifica y refuerza la regulación y supervisión del sistema por el Estado; y

» establece una base financiera sólida para la re-reforma basada en un fondo de reserva solidario, proyecciones actuariales cada tres años, evaluaciones actuariales cada cinco años del efecto de diversas variables sobre elementos clave de las pensiones, y el monitoreo por un consejo asesor técnico y la comisión de usuarios. 


\subsection{Problemas y retos ${ }^{5}$}

» Implementar mecanismos más innovadores y eficaces para lograr la cobertura obligatoria de los trabajadores autónomos; a pesar de un incremento, los afiliados son todavía muy pocos en relación con el número total de los autónomos; lo mismo puede decirse de la afiliación obligatoria de las empleadas domésticas, así como de las afiliaciones de trabajadores voluntarios y de los jóvenes;

" eliminar el pequeño porcentaje de "polizontes" a través de una aplicación más eficaz y verificación periódica del nuevo mecanismo de focalización, hacer transparentes las causas de la suspensión de pensiones;

» investigar el impacto real de las pensiones solidarias sobre la afiliación y el pago de las cotizaciones al sistema contributivo, así como sobre el ahorro;

» integrar los regímenes separados de pensiones de las fuerzas armadas y de la policía en el sistema general, y eliminar o reducir los subsidios fiscales concedidos a sus miembros;

» enfrentar las desigualdades de género generadas por el mercado de trabajo a través de medios legales (aplicación de igual salario a mujeres y hombres por el mismo trabajo), pero también fomentar la mayor participación laboral femenina a través de la capacitación y una mayor extensión de las guarderías infantiles públicas;

» estudiar la viabilidad de sustituir las tablas de mortalidad diferenciadas por sexo, por tablas unisexo;

» investigar la reclamación de los sindicatos de que las pensiones contributivas son todavía muy bajas (ver 4);

» persiste en la población el escaso conocimiento del sistema de pensiones, la información disponible es muy técnica y no ajustada el nivel educativo de la mayoría del pueblo por lo que debería simplificarse y la población debería ser educada mejor a través del fondo de educación previsional, el cual debe difundir adecuadamente los nuevos derechos;

" los "multifondos" ayudaron a proteger a la mayoría de los asegurados durante la crisis global, pero la información proporcionada por la AFP y los asesores privados aún debe mejorarse para lograr una mejor selección de carteras y cambios a través del tiempo;

» para mejorar aún más el pago de las cotizaciones, el asegurado debe desempeñar un papel más activo en la detección de la mora del empleador verificando el informe trimestral enviado por la AFP, y esta notificar sin demora a los trabajadores de dicho retraso; $y$

» continuar los informes anuales del CPS y del CUSP, así como las evaluaciones actuariales estipuladas por la ley para asegurar que se asignen los fondos necesarios en el presupuesto destinados al pago de las nuevas prestaciones.

\footnotetext{
${ }^{5}$ Esta sección se basa principalmente en CPS, 2011 y CUSP, 2011.
} 


\section{Epílogo}

Durante la campaña electoral a la Presidencia de Chile en 2013 surgió un debate público sobre el sistema de pensiones reformado por Michelle Bachelet en 2008 y ella incluyó en su programa la creación de una AFP pública para estimular la competencia y bajar los costos. Pero el debate tocó otros dos puntos críticos: a) se alega que las tasas de reemplazo nacionales de las pensiones promedian 54,5\% del último salario para los hombres y $44,1 \%$ para las mujeres (esto se basa en el último salario antes del retiro en vez del promedio salarial durante todo el período activo del trabajador; la tasa mínima recomendada por la OIT es $45 \%$ del salario base); y b) la edad estatutaria de retiro en Chile es 65 años para ambos sexos (el asegurado puede retirarse a menor edad siempre que tenga cierta suma acumulada en la cuenta individual) pero la edad efectiva de retiro promedio para los hombres es de 69,4 años y para las mujeres es 70,4 años, lo cual indica que los asegurados deben trabajar más tiempo a fin de lograr una acumulación mayor en sus cuentas individuales. Una posición extrema aboga por la substitución del sistema privado por uno público de reparto, otra más moderada sugiere la conversión del sistema privado en mixto con un período de transición. Se plantea la necesidad de un referendo nacional para que la población determine qué sistema quiere, así como una comisión de reforma amplia que incluya las organizaciones sociales, los asegurados y expertos. La Presidenta Bachelet todavía no se ha pronunciado sobre estas propuestas (Carmona y Fernández, 2013).

\section{Bibliografia}

Arenas de Mesa, A. (2010): Historia de la Reforma Previsional Chilena: Una Experiencia Exitosa de Política Pública en Democracia, Santiago, OIT.

Arenas de Mesa, A. (2012): Información suministrada al autor, Santiago, agosto 30.

Arenas de Mesa, A. et al. (2008): La Reforma Previsional Chilena: Proyecciones Fiscales 2009-2025, Santiago, Gobierno de Chile, Estudios de Finanzas Públicas.

Asociación Internacional de Organismos de Supervisión de Fondos de Pensiones - AiOS (1999 a 2011): Boletín Estadístico AIOS, 1 a 24, diciembre.

Asociación Internacional de la Seguridad Social - AISS. (2011, 2012): Observador de la Seguridad Social, agosto y enero.

Banco Mundial (1994): Envejecimiento sin Crisis, Oxford, Oxford University Press.

Berhman, J. et al. (2011): "First Round Impact of the 2008 Chilean Pension System Reform", Working Paper 245, Ann Arbor, University of Michigan Retirement Research Center.

Carmona, A. y B. Fernández (2013): "El plan de transición que se discute en el comando de Bachelet para poner fin a las AFP", El Mostrador, Santiago, 6 diciembre. 
Comisión de Usuarios del Sistema de Pensiones - CUSP (2011): Segundo Informe Agosto 2011, Santiago, Subsecretaría de Previsión Social.

Comisión Económica para América Latina y el CARibe - CEPAL (2011): Balance Preliminar de las Economías de América Latina y el Caribe 2011, Santiago, diciembre.

Consejo Consultivo Previsional - CCP (2011): Informe Anual: Segundo Informe Enero 2010-Julio 2011, Santiago, Subsecretaría de Previsión Social.

Federación Internacional de Administradoras de Fondos de Pensiones-FiaP (2011): Informe Trimestral No. 31, 30.06.2011, Santiago, junio.

Fultz, E. (2012): “The Retrenchment of Second-Tier Pensions in Hungary and Poland: A Precautionary Tale", International Social Security Review, 65 (3), pp.1-25.

Gobierno de Chile (2008): “Ley 20,255”, Diario Oficial, Santiago, 17 marzo.

Instituto Nacional de Estadísticas - INE (2012): Chile Encuesta Nacional de Hogares (varios años).

Mesa-Lago, C. (2008a): Reassembling Social Security: A Survey of Pension and Health Care Reforms in Latin America, Oxford, Oxford University Press.

Mesa-Lago, C. (2008b): "Protección Social en Chile: Reformas para Mejorar la Equidad", Revista Internacional del Trabajo, 147 (4), pp. 421-446.

Mesa-Lago, C. (2010): World Crisis Effects on Social Security in Latin American and the Caribbean: Lessons and Policies, Londres, Institute for the Study of the Americas.

Mesa-Lago, C. (2012): "Re-Reforms of Privatized Pensions Systems",monografia de Zeitschrift für ausländisches und internationales Arbeits- und Sozialrecht, 26, (3), pp.189-316.

Mesa-Lago, C. (2013): "Privatisation of Chile's Pension and Health Care Systems", en Frans Pennings, Ulrik Becker y Tineke Dijkhoff, (comps.), International StandardSetting and Innovations in Social Security, Wolters Kluwer International, pp. 387-413.

MülLer, K. (2003): Privatising Old-Age Security: Latin American and Eastern Europe Compared, Cheltenhalm, E. Edgar.

Rofman, R. y M. L. Oliveri (2012): 'Pension Coverage in Latin America: Trends and Determinats", Social Protection and Labor Discussion Paper, 1217, Washington D.C., Banco Mundial.

Rofman, R. y L. Lucchetti (2007): "Pensions Systems in Latin America: Concepts and Measurements of Coverage", SP Discussion Paper 616, Washington D.C., Banco Mundial. Superintendencia de AFP - SAFP (1981 a 2009): Boletín Estadístico, 1 a 207, Santiago.

Superintendencia de Pensiones (SP) $(2011,2012)$ : Centro de Estadísticas, diciembre y julio, Disponible en:

$<$ www.spensiones.cl> (consulta: 21 de septiembre de 2015). 